\title{
Proximal ligation after the side-to-end anastomosis recovery technique for lymphaticovenous anastomosis
}

\author{
Yushi Suzuki ${ }^{1,2}$, Hisashi Sakuma ${ }^{2}$, Jun Ihara ${ }^{3}$, Yusuke Shimizu ${ }^{1}$ \\ ${ }^{1}$ Department of Plastic and Reconstructive Surgery, University of the Ryukyus, Nishihara; ${ }^{2}$ Department of Plastic and Reconstructive Surgery, \\ Yokohama Municipal Citizen's Hospital, Yokohama; ${ }^{3}$ Department of Plastic and Reconstructive Surgery, Sano Kosei General Hospital, Sano, \\ Japan
}

Background Lymphaticovenous anastomosis is an important surgical treatment for lymphedema, with lymphaticovenous side-to-end anastomosis (LVSEA) and lymphaticovenous endto-end anastomosis being the most frequently performed procedures. However, LVSEA can cause lymphatic flow obstruction because of regurgitation and tension in the anastomosis. In this study, we introduce a novel and simple procedure to overcome this problem.

Methods Thirty-five female patients with lower extremity lymphedema who underwent lymphaticovenous anastomosis at our hospital were included in this study. Eighty-five LVSEA procedures were performed, of which 12 resulted in insufficient venous blood flow. For these 12 anastomoses, the proximal lymphatic vessel underwent clipping after the anastomotic procedure and the venous inflow was monitored. Subsequently, the proximal ligation after side-to-end anastomosis recovery (PLASTER) technique, which involves ligating the proximal side of the lymphatic vessel, was applied. A postoperative evaluation was performed using indocyanine green 6 months after surgery.

Results Despite the clipping procedure, three of the 12 anastomoses still showed poor venous inflow. Therefore, it was not possible to apply the PLASTER technique in those cases. Among the nine remaining anastomoses in which the PLASTER technique was applied, three (33\%) were patent.

Conclusions Our findings show that achieving patent anastomosis is challenging when postoperative venous inflow is poor. We achieved good results by performing proximal ligation after LVSEA. Thus, the PLASTER technique is a particularly useful recovery technique when LVSEA does not result in good run-off.
Correspondence: Yushi Suzuki Department of Plastic and Reconstructive Surgery, University of the Ryukyus, Uehara 207, Nishiharacho, Nakagami-gun, Okinawa 9030125, Japan

Tel: +81-98-895-3331

Fax: +81-98-895-1090

E-mail: yushisuzuki-kei@umin.ac.jp

Keywords Microsurgery / Lymphatic vessels / Edema / Lymphatic diseases

Received: 20 Nov $2018 \bullet$ Revised: 15 Jun $2019 \bullet$ Accepted: 21 Jun 2019

pISSN: 2234-6163 • elSSN: 2234-6171 • https://doi.org/10.5999/aps.2018.01382・ Arch Plast Surg 2019;46:344-349

\section{INTRODUCTION}

Lymphaticovenous anastomosis (LVA), the mainstay surgical treatment for cases of lymphedema, provides symptom relief by creating an anastomosis between the congested lymphatic vessel (owing to lymphedema) and a vein. Because the diameter of 
the lymphatic vessel is usually smaller than $0.8 \mathrm{~mm}$, supermicrosurgery is warranted [1].

Lymphaticovenous side-to-end anastomosis (LVSEA) and lymphaticovenous end-to-end anastomosis (LVEEA) are the two most frequently used procedures for anastomoses [2]; however, no previous reports have shown a significant difference between these methods. In addition, the postoperative patency rate after LVA is not high [3-5]. Therefore, we selected LVSEA as the principal method, considering the risk of occlusion. However, in cases of LVSEA, we sometimes face difficulty in establishing lymphatic flow into the vein after anastomosis because of venous regurgitation. To solve this problem, we ligated the proximal side of the lymphatic vessel, which resulted in improved inflow into the vein.

\section{METHODS}

\section{Operative technique}

\section{Conventional LVSEA}

We performed LVA surgery under local or general anesthesia. The selection of anesthesia was based on the patient's choice, except in cases where the patient's general condition made it difficult to use general anesthesia. Using indocyanine green (ICG) and PDE-neo (Hamamatsu Photonics, Hamamatsu, Japan), we assessed the lymphatic vessel the day before surgery. We then identified the vein to be anastomosed using ultrasonography near the lymphatic vein. Following this image, we determined the skin incisions that would be used to access both the vein and lymphatic vessel running near the site.

During the operation, we dyed the lymphatic vessel using pat- ent blue (Wako Junyaku Kogyo Co., Ltd., Osaka, Japan) [6] to make detection easier. If we observed good lymphatic vessel flow, LVSEA was performed primarily to preserve the patient's lymphatic flow. The lateral wall of the lymphatic vessel was cut using an ophthalmic surgical knife, and suturing was performed using 12-0 nylon. After anastomosis, the lymphatic flow run-off was determined by testing the vein patency using two forceps. Excellent run-off was obtained if the flow was smooth without any compression, whereas good run-off was obtained with mild compression. However, if the inflow into the vein was low, even with intense compression of the surrounding tissue, or if regurgitation of the vein flow was high, we classified the anastomosis as poor (Table 1$)$.

\section{PLASTER technique}

This method was selected when the LVSEA run-off was poor (Fig. 1). We clipped the proximal lymphatic vessel temporarily in cases where the flow was unsatisfactory after anastomosis. If the inflow to the vein increased after temporary clipping, we ap-

\section{Table 1. Run-off classification [4]}

\begin{tabular}{|ll|}
\hline Run-off & $\begin{array}{c}\text { Classification of run-off depending on lymphatic } \\
\text { inflow after anastomosis }\end{array}$ \\
\hline $\begin{array}{l}\text { Excellent } \\
\text { Good }\end{array}$ & $\begin{array}{c}\text { The flow is smooth without compression by lymphatic function. } \\
\text { The inflow to the vein is sufficient with mild compression of the } \\
\text { surrounding tissues. } \\
\text { There is flow into the vessel with compression surrounding the } \\
\text { Fair }\end{array}$ \\
tissues. \\
There is poor inflow into the vein even with compression of the \\
surrounding tissue.
\end{tabular}

\section{Fig. 1. Obstructed lymphatic flow because of venous regurgitation}

While we perform precise anastomosis, we sometimes experience poor lymphatic flow to the vein due to venous regurgitation. We use patent blue dye during the operation to find lymphatic vessels easily. Hence, the color of the lymphatic vessel is blue.
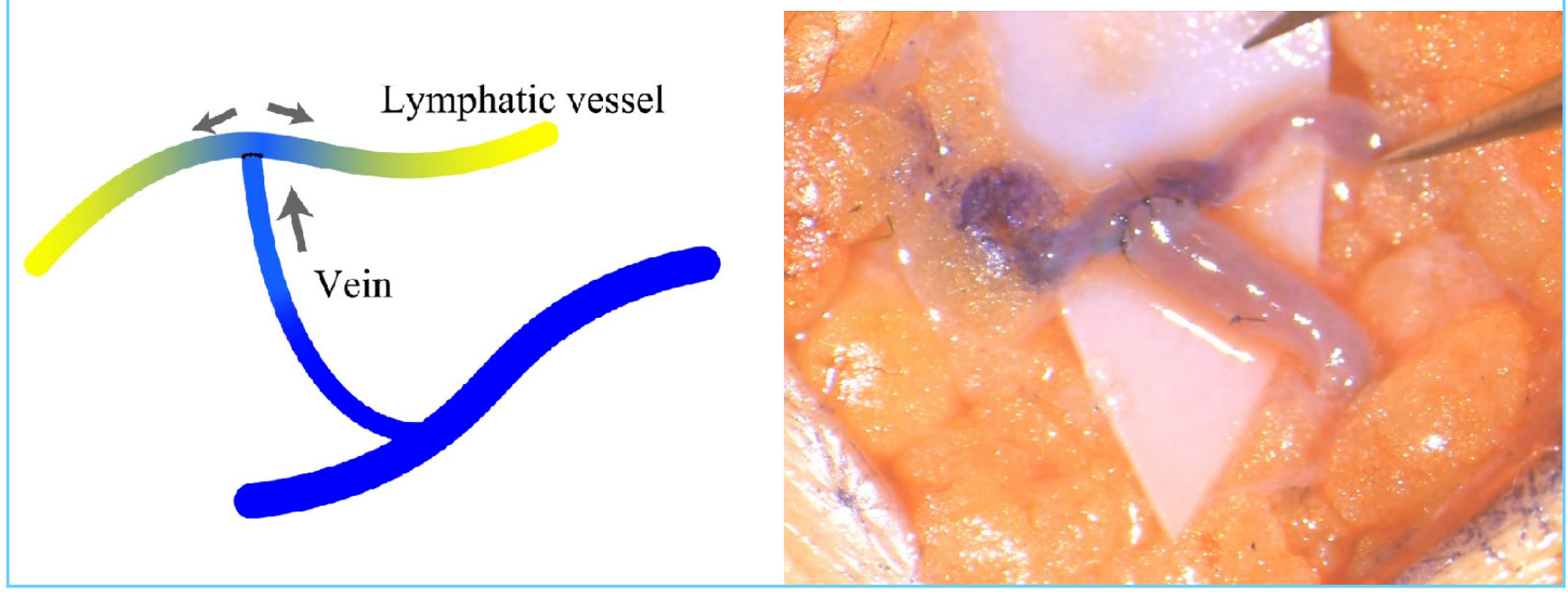


\section{Fig. 2. Temporary clipping of the proximal side}

After clipping the proximal side of the lymphatic vessel, the vein was filled with blue liquid, suggesting good inflow to the vein. We use patent blue dye during the operation to find lymphatic vessels easily. Hence, the color of the lymphatic vessel is blue.
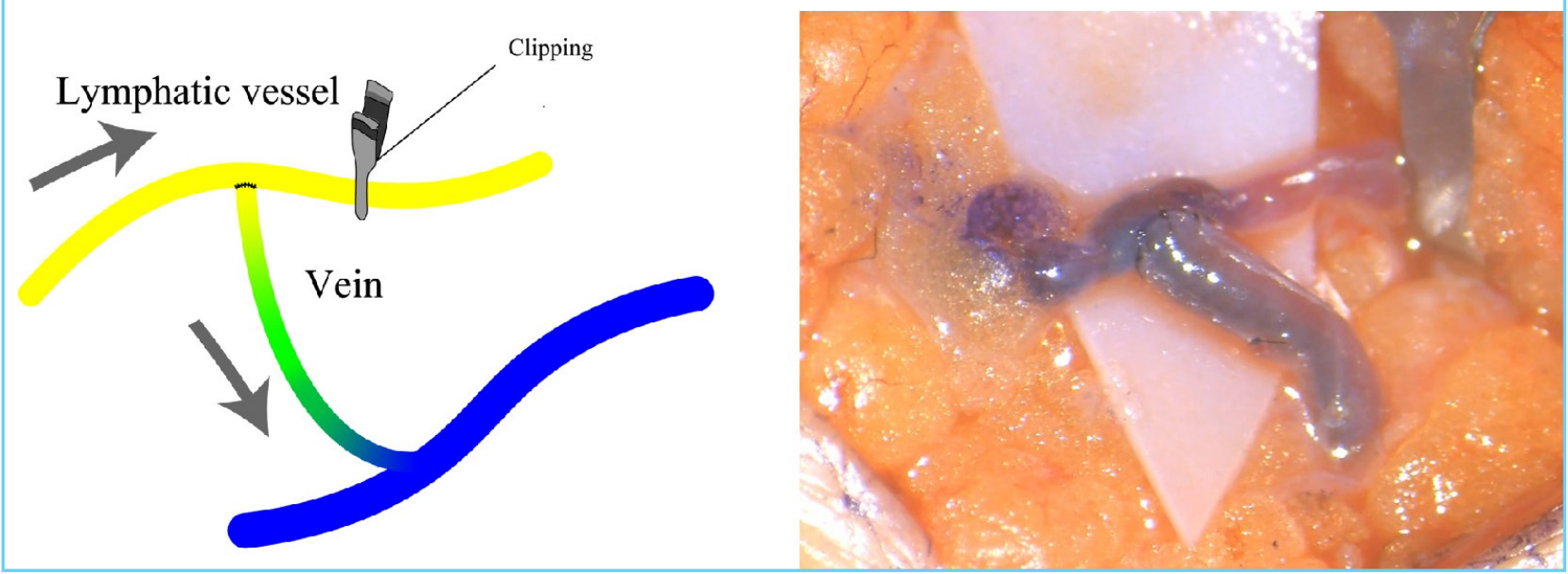

\section{Fig. 3. Proximal side ligation of the lymphatic vessel}

After the ligation, the vein was filled with blue liquid, suggesting high lymphatic flow and excellent run-off. We use patent blue dye during the operation to find lymphatic vessels easily. Hence, the color of the lymphatic vessel is blue.
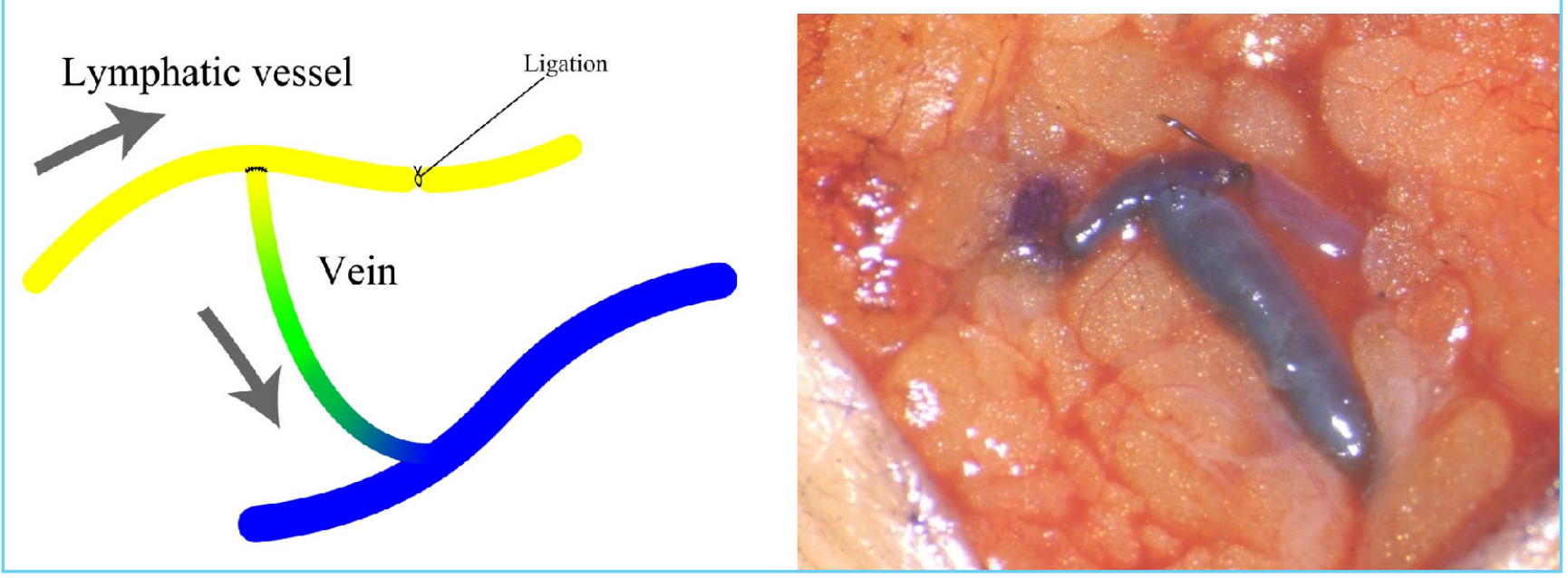

plied the proximal ligation after side-to-end anastomosis recovery (PLASTER) technique (Fig. 2). If inflow to the vein was sufficient under mild compression after clipping, good lymphatic run-off was likely to be obtained after ligating the proximal side of the lymphatic vessel using $12-0$ nylon (Fig. 3 ). The lymphatic vessel was not cut after ligation. The PLASTER technique was not applicable when inflow to the vein was poor after clipping; thus, in such cases, the wound was closed (Supplemental Video 1).

\section{Postoperative evaluation}

Intermittent pneumatic compression was applied 2 days after the operation. Thereafter, the patients wore the same compres- sion garments as those used preoperatively until the ICG test around 6 months after the operation. The postoperative evaluation was conducted using ICG 6 months after the LVA operation. After injecting ICG into the subcutaneous tissue, we visualized the anastomosis point using PDE-neo. The patency of the anastomosis was assessed.

Patients' medical records, intraoperative pictures, and motion videos were retrospectively reviewed. The study was performed in accordance with the principles of the Declaration of Helsinki. All patients in this study provided written (signed) informed consent for the use of preoperative, intraoperative, and postoperative data for academic publication without disclosure of their personal information. 


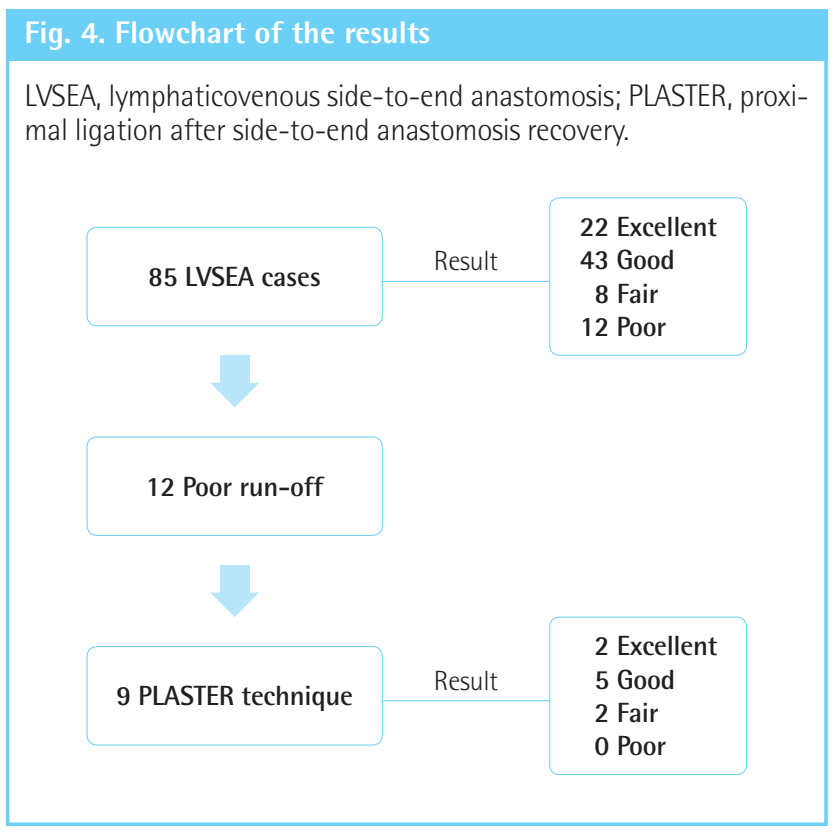

\section{Cases}

Thirty-five female patients with lower extremity lymphedema who underwent LVA at Yokohama Municipal Citizen's Hospital were included in this study. Eighty-five LVSEA procedures were performed, of which 12 anastomoses resulted in poor run-off. Nine of those 12 anastomoses were treated using the PLASTER technique (Fig. 4).

\section{RESULTS}

Among the 85 LVSEA procedures, 22 resulted in excellent outcomes, 43 resulted in good outcomes, eight resulted in fair outcomes, and 12 resulted in poor run-off. For the 12 LVSEA cases with poor run-off, we considered applying the PLASTER technique. Of the cases in which the PLASTER technique was performed, two showed excellent outcomes, five had good outcomes, and two had fair outcomes. Despite the clipping procedure, three of the 12 LVSEA cases continued to show poor runoff. Thus, it was not possible to apply the PLASTER technique in those cases (Fig. 4).

Among the nine cases that underwent the PLASTER technique, three (33\%) had patent anastomoses. In contrast, the three cases in which the PLASTER technique was not used, owing to poor postoperative run-off, did not show patent anastomoses.

\section{DISCUSSION}

Surgical treatments, such as LVA and lymph node transfer, are indicated for the treatment of lymphedema, in combination with conservative compression therapy $[7,8]$. Physiological lymph flow can be reestablished effectively by connecting the congested lymphatic vessel to a vein. This surgical procedure is relatively noninvasive because the skin incision is only $3 \mathrm{~cm}$ long, and studies have reported favorable results for the treatment of lymphedema [3,8-10].

We sought to determine the benefits of this procedure by observing lymphatic flow and patency directly. Very few previous studies have evaluated anastomoses directly using ICG; however, this evaluation technique enabled us to directly observe the surgical results. Occlusion of the anastomosis site will occur even if an excellent anastomosis is obtained [3-5]. Therefore, we selected LVSEA as the principal method. We believe that the proximal side of the lymphatic flow can be preserved even if occlusion occurs. However, occasionally, lymphatic inflow to the vein does not occur, even if LVSEA is performed accurately, because of the venous pressure and angle of the anastomosis. Venous regurgitation occurs sometimes in LVA surgery. To overcome this complication, several techniques via the venous approach have been reported $[11,12]$. Intraoperative and postoperative compressions are also among the approaches used to increase lymphatic flow and to improve anastomosis, thereby contributing to good results $[13,14]$. At our institution, if the inflow of lymph fluid to the blood vessel could be obtained by using some level of compression, we considered the created shunt as likely to be effective because postoperative compression would be applied. However, poor run-off or poor inflow into the vein even with compression of the surrounding tissue, based on our criteria for anastomosis, would be indicative of an ineffective shunt even if postoperative compression was applied. In such cases, we used the PLASTER technique, which creates a dam of lymph fluid that increases the amount and pressure of the lymph. Our technique is an approach to overcome this issue. Moreover, this technique has the advantage of temporary clamping of the proximal side, as we can decide whether to ligate after checking the lymphatic flow. Thus, if inflow to the vein is poor even after clamping the lymphatic vessel, the lymphatic flow is preserved by declamping. Postoperative patency is highly limited if the run-off is poor [4]. Our results also show that in cases involving poor run-off, patency could not be achieved 6 months after the operation. Therefore, if an operation results in poor flow, it is highly possible that the postoperative results will be poor. However, if good venous inflow can be achieved using the PLASTER technique, part of the LVSEA can be recovered with this simple procedure. Thus, this technique is simple, but provides a sophisticated result. It is important to try to achieve postoperative patency because postoperative patency and symptom relief are strongly correlated [5]. To achieve good 
results, it is important to obtain good run-off.

We hypothesize that lymphatic reconnection is easier when ligating the lymphatic vein alone, rather than cutting it off, because an occluded lymphatic vessel can regenerate spontaneously and create a collateral pathway [15]. This may be gentler on the lymphatic flow than LVEEA, which cuts off the lymphatic vessel completely.

We presented cases that showed good venous inflow in response to ligation of the proximal side of the lymphatic vessel. This study is limited by its small sample size, but our results suggest that the PLASTER technique can be an extremely useful recovery technique if it is difficult to obtain good inflow after LVSEA. Future studies should be conducted on larger groups of patients.

\section{NOTES}

\section{Conflict of interest}

No potential conflict of interest relevant to this article was reported.

\section{Ethical approval}

The study was performed in accordance with the principles of the Declaration of Helsinki. Written informed consents were obtained.

\section{Patient consent}

The patients provided written informed consent for the publication and the use of their images.

\section{Author contribution}

Conceptualization: Suzuki Y, Sakuma H. Data curation: Suzuki Y, Sakuma H, Ihara J, Shimizu Y. Formal analysis: Suzuki Y. Methodology: Suzuki Y, Sakuma H, Ihara J. Project administration: Suzuki Y, Sakuma H. Visualization: Suzuki Y. Writing original draft: Suzuki Y. Writing - review \& editing: Suzuki Y. Approval of final manuscript: all authors.

\section{ORCID}

Yushi Suzuki https://orcid.org/0000-0003-1674-4090

Hisashi Sakuma https://orcid.org/0000-0002-6073-6832

Jun Ihara https://orcid.org/0000-0001-8824-308X

Yusuke Shimizu https://orcid.org/0000-0002-2528-2003

\section{Supplementary material}

Supplemental Video 1. The proximal ligation after side-to-end anastomosis recovery (PLASTER) technique. This video shows significant improvement of lymphatic inflow after the clipping, which is a good indication of the PLASTER technique. Supplemental data can be found at: https://doi.org/10.5999/ aps.2018.01382.v001.

\section{REFERENCES}

1. Masia J, Olivares L, Koshima I, et al. Barcelona consensus on supermicrosurgery. J Reconstr Microsurg 2014;30:53-8.

2. Cheng MH, Chang DW, Patel KM. Principles and practice of lymphedema surgery. Edinburgh: Elsevier; 2016.

3. Maegawa J, Yabuki Y, Tomoeda H, et al. Outcomes of lymphaticovenous side-to-end anastomosis in peripheral lymphedema. J Vasc Surg 2012;55:753-60.

4. Suzuki Y, Sakuma H, Yamazaki S. Evaluation of patency rates of different lymphaticovenous anastomosis techniques and risk factors for obstruction in secondary upper extremity lymphedema. J Vasc Surg Venous Lymphat Disord 2019;7: 113-7.

5. Suzuki Y, Sakuma H, Yamazaki S. Comparison of patency rates of lymphaticovenous anastomoses at different sites for lower extremity lymphedema. J Vasc Surg Venous Lymphat Disord 2019; 7:222-7.

6. Yap YL, Lim J, Shim TW, et al. Patent blue dye in lymphaticovenular anastomosis. Ann Acad Med Singapore 2009;38: 704-6.

7. Ozturk CN, Ozturk C, Glasgow M, et al. Free vascularized lymph node transfer for treatment of lymphedema: a systematic evidence based review. J Plast Reconstr Aesthet Surg 2016;69:1234-47.

8. Scaglioni MF, Fontein DB, Arvanitakis M, et al. Systematic review of lymphovenous anastomosis (LVA) for the treatment of lymphedema. Microsurgery 2017;37:947-53.

9. Campisi C, Boccardo F, Zilli A, et al. Long-term results after lymphatic-venous anastomoses for the treatment of obstructive lymphedema. Microsurgery 2001;21:135-9.

10. O’Brien BM, Mellow CG, Khazanchi RK, et al. Long-term results after microlymphaticovenous anastomoses for the treatment of obstructive lymphedema. Plast Reconstr Surg 1990;85:562-72.

11. Akita S, Mitsukawa N, Kuriyama M, et al. External valvuloplasty for subcutaneous small veins to prevent venous reflux in lymphaticovenular anastomosis for lower extremity lymphedema. Plast Reconstr Surg 2013; 132:1008-14.

12. Yamamoto T, Giacalone G. Lymphatic vessel grafting for prevention of venous reflux into a sclerotic lymphatic vessel in supermicrosurgical lymphaticovenular anastomosis. J Plast Reconstr Aesthet Surg 2016;69:e67-8.

13. Fuse Y, Yamamoto T. Intraoperative distal compression in 
supermicrosurgical lymphaticovenous anastomosis for lymphedema. J Surg Oncol 2018;118:243-4.

14. Chen WF, Bowen M, Ding J. Immediate limb compression following supermicrosurgical lymphaticovenular anastomo- sis-is it helpful or harmful? Int Microsurg J 2018;2:1.

15. Yan A, Avraham T, Zampell JC, et al. Mechanisms of lymphatic regeneration after tissue transfer. PLoS One 2011;6: e17201. 